\title{
Transport reversal in a thermal ratchet
}

\author{
Baoquan Ai, ${ }^{1,2}$ Liqiu Wang, ${ }^{1}$ and Lianggang $\mathrm{Liu}^{3}$ \\ ${ }^{1}$ Department of Mechanical Engineering, The University of Hong Kong, Pokfulam Road, Hong Kong \\ ${ }^{2}$ School of Physics and Telecommunication Engineering, South China Normal University, GuangZhou, China \\ ${ }^{3}$ Department of Physics, ZhongShan University, GuangZhou, China
}

(Received 24 April 2005; published 8 September 2005)

\begin{abstract}
Transport of a Brownian particle moving in a periodic potential is investigated in the presence of a symmetric unbiased external force. The viscous medium is alternately in contact with two heat reservoirs. We present the analytical expression of the net current at the quasi-steady-state limit. It is found that the competition of the asymmetric parameter of the potential with the temperature difference leads to phenomena like current reversal. The competition between the two driving factors is a necessary but not a sufficient condition for current reversals.
\end{abstract}

DOI: 10.1103/PhysRevE.72.031101

PACS number(s): 05.40.- a, 05.70.- a, 87.10.+e

\section{INTRODUCTION}

Transport phenomena play a crucial role in many processes from physical and biological to social systems. There has been increasing interest in transport properties of nonlinear systems which can extract usable work from unbiased nonequilibrium fluctuations [1-4]. This comes from the desire to understand molecular motors [5], nanoscale friction [6], surface smoothing [7], coupled Josephson junctions [8], optical ratchets and directed motion of laser-cooled atoms [9], and mass separation and trapping schemes at the microscale [10].

The focus of research has been on noise-induced unidirectional motion over the last decade. A ratchet system is generally defined as a system that is able to transport particles in a periodic structure with nonzero macroscopic velocity in the absence of macroscopic force on average. In these systems, directed Brownian motion of particles is generated by nonequilibrium noise in the absence of any net macroscopic forces and potential gradients. Typical examples are rocking ratchets $[4,11]$, flashing ratchets [12], diffusion ratchets [13], correlation ratchets $[4,14]$, and white-shot-noise ratchets [2]. In all these studies, the potential is taken to be asymmetric in space. It has also been shown that a unidirectional current can also appear for spatially symmetric potentials if there exists an external random force either asymmetric or spatially dependent. If spatially periodic structures are exposed to additive Poissonian white shot noise of zero average, a macroscopic current occurs even in the absence of spatial asymmetry [2].

The current reversal is very important in new particle separation devices such as electrophoretic separation of microparticles [15]. It is also of interest in biology [32]. Motions of macromolecules are probably responsible for the vesicle transport inside eukaryotic cells. A typical example is the motion of proteins along a microtubule, modeled usually by a ratchet [29]. It is well known that the two typical proteins kinesins and dyneins move along tubulin filaments in opposite directions. This can be explained by the current reversal.

Current reversal in ratchet systems can be engendered by varying the system parameters [16-28]. The current can be reversed, for example, by a noise of Gaussian force with a nonwhite power spectrum in the presence of a stationary periodic potential [19]. The current reversal can also be obtained in two-state ratchets if the long arm is kinked [20]. Bier and Astumian [21] have also found current reversal in a fluctuating three-state ratchet. In the presence of a kangaroo process as the driving force, the current reversal can be triggered by varying the noise flatness, the ratio of the fourth moment to the square of the second moment [22]. The current reversal can be induced by both an additive Gaussian white and an additive Ornstein-Uhlenbeck noise in a correlation ratchet [23]. The current reversal also appears in forced inhomogeneous ratchets $[17,18]$.

The previous works on current reversal are limited to the case of one heat reservoir. The present study extends the study of current reversal to the case of two heat reservoirs. When a positive driving factor competes with a negative one, the current may reverse its direction. The competition between the competitive driving factors is necessary but not sufficient for the current reversal. Our emphasis is on finding the conditions that generate current reversal. This is achieved by using a quasi-steady-state limit to solve the FokkerPlanck equation.

\section{NET CURRENT OF THE THERMAL RATCHET}

Consider a Brownian particle moving in a sawtooth potential with an unbiased external force where the medium is alternately in contact with two heat reservoirs. This model was first proposed to describe molecular motor in biological systems [29]. The particle motion satisfies the dimensionless Langevin equation of motion [30,31]

$$
m \frac{d^{2} x}{d t^{2}}=-\beta \frac{d x}{d t}-\frac{d U(x)}{d x}+F(t)+\sqrt{2 k_{B} T(x) \beta} \xi(t),
$$

where $x$ stands for the position of the Brownian particle, $m$ the mass of the particle, $\beta$ the viscous friction drag coefficient, $k_{B}$ the Boltzmann constant, and $T(x)$ the absolute temperature. $\xi(t)$ is a randomly fluctuating Gaussian white noise of zero mean and the autocorrelation function 
$\langle\xi(t) \xi(s)\rangle=\delta(t-s)$. Here $\langle\cdots\rangle$ denotes an ensemble average over the distribution of the fluctuating forces $\xi(t) . F(t)$ is an external periodic force [Fig. 1(b)], satisfying

$$
F(t+\tau)=F(t), \quad \int_{0}^{\tau} F(t) d t=0 .
$$

The geometry of the symmetric potential $U(x)=U(x+L)$ is displayed in Fig. 1(a) and $U(x)$ within the interval $0 \leqslant x$ $\leqslant L$ is described by

$$
U(x)= \begin{cases}\frac{U}{L_{1}} x, & 0 \leqslant x<L_{1}, \\ \frac{U}{L_{2}}(L-x), & L_{1} \leqslant x \leqslant L,\end{cases}
$$

where $L=L_{1}+L_{2}$ is the period of the potential. The temperature $T(x)$ has the same period as the potential $U(x)$. Therefore, $T(x)=T(x+L)$,

$$
T(x)= \begin{cases}T+\delta, & 0 \leqslant x<L_{1}, \\ T, & L_{1} \leqslant x \leqslant L .\end{cases}
$$

Because the motion of the ratchet is highly overdamped in general [30], the inertia term can be neglected. Hence, Eq. (1) reduces, when $\beta=1$ and $k_{B}=1$, to

$$
\frac{d x}{d t}=-\frac{d U(x)}{d x}+F(t)+\sqrt{2 T(x)} \xi(t) .
$$

The probability density satisfies the associated FokkerPlanck equation $[30,31]$

$$
\begin{aligned}
\frac{\partial P(x, t)}{\partial t} & =\frac{\partial}{\partial x}\left(\left[U^{\prime}(x)-F(t)\right] P(x, t)+\frac{\partial}{\partial x}[T(x) P(x, t)]\right) \\
& =-\frac{\partial j(x, t)}{\partial x}, \\
j(x, t) & =-\left[U^{\prime}(x)-F(t)\right] P(x, t)-\frac{d}{d x}[T(x) P(x, t)] ;
\end{aligned}
$$

here $j$ is the probability current density. The prime stands for the derivative with respect to the space variable $x . P(x, t)$ is the probability density for the particle at position $x$ and at time $t$. It satisfies the normalization condition and the periodicity condition,

$$
\begin{gathered}
P(x, t)=P(x+L, t), \\
\int_{0}^{L} P(x, t) d x=1 .
\end{gathered}
$$

If $F(t)$ changes very slowly with respect to $t$, namely, its period is longer than any other time scale of the system, there exists a quasi-steady-state. In this case, by following the method in [30,31], we can obtain the current $j(F(t))$ from Eqs. (7)-(9),
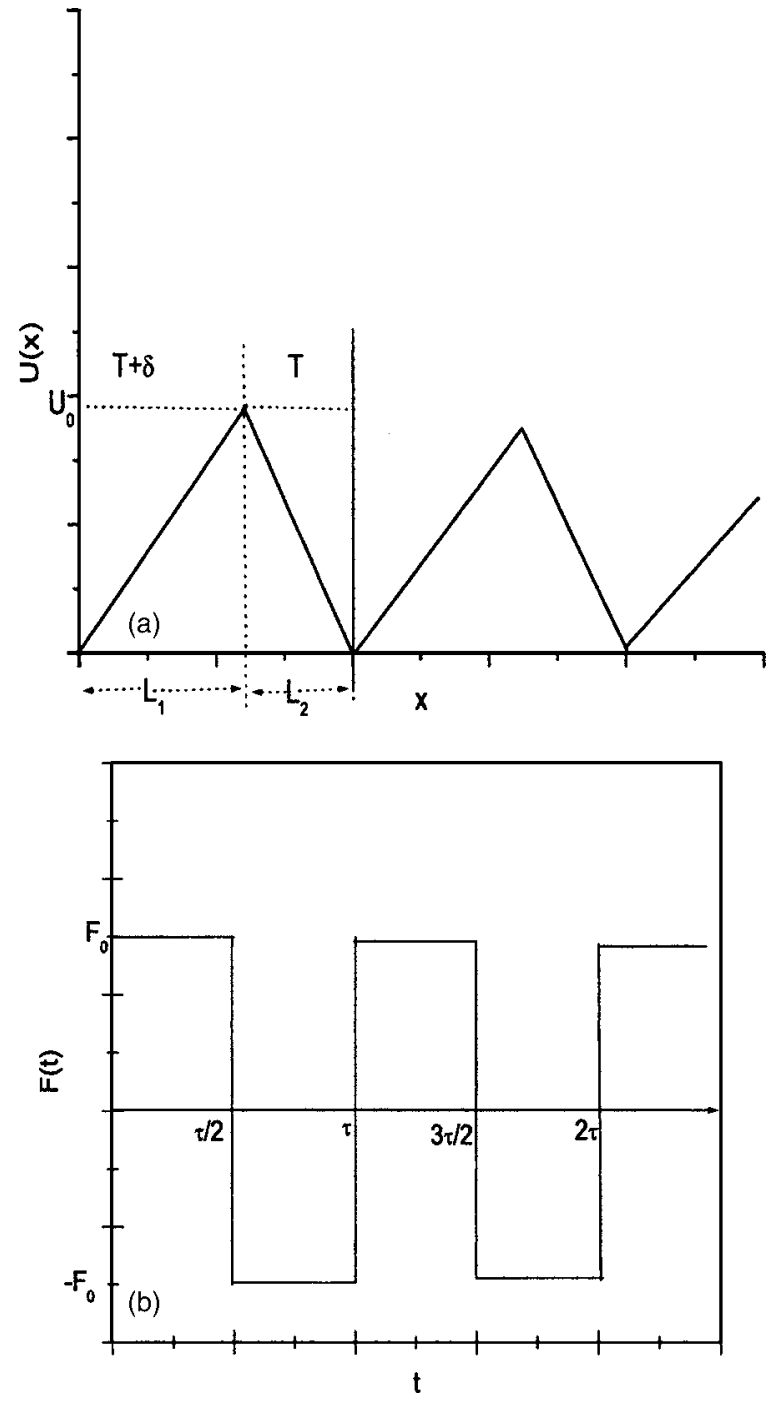

FIG. 1. Potential and driving force. (a) Potential $U(x)$ $=U(x+L) ; U(x)$ is a piecewise linear and periodic potential; the period of the potential is $L=L_{1}+L_{2} ; \Delta=L_{1}-L_{2}$; the temperature profile is also shown. (b) Driving force $\mathrm{F}(\mathrm{t})$ which preserved the zero mean $\langle F(t)\rangle=0 ; F(t+\tau)=F(t) ; F_{0}$ is amplitude of $F(t)$.

$$
j(F(t))=\frac{-Q}{G_{1} G_{2}+H Q},
$$

where $Q, G_{1}, G_{2}$, and $H$ are

$$
Q=e^{a-b}-1,
$$

$$
\begin{gathered}
G_{1}=\frac{L+\Delta}{2 a(T+\delta)}\left(1-e^{-a}\right)+\frac{L-\Delta}{2 b T} e^{-a}\left(e^{b}-1\right), \\
G_{2}=\frac{L+\Delta}{2 a}\left(e^{a}-1\right)+\frac{L-\Delta}{2 b} e^{a}\left(1-e^{-b}\right), \\
H=A+B+C, \\
A=\frac{1}{T+\delta}\left(\frac{L+\Delta}{2 a}\right)^{2}\left(a+e^{-a}-1\right),
\end{gathered}
$$




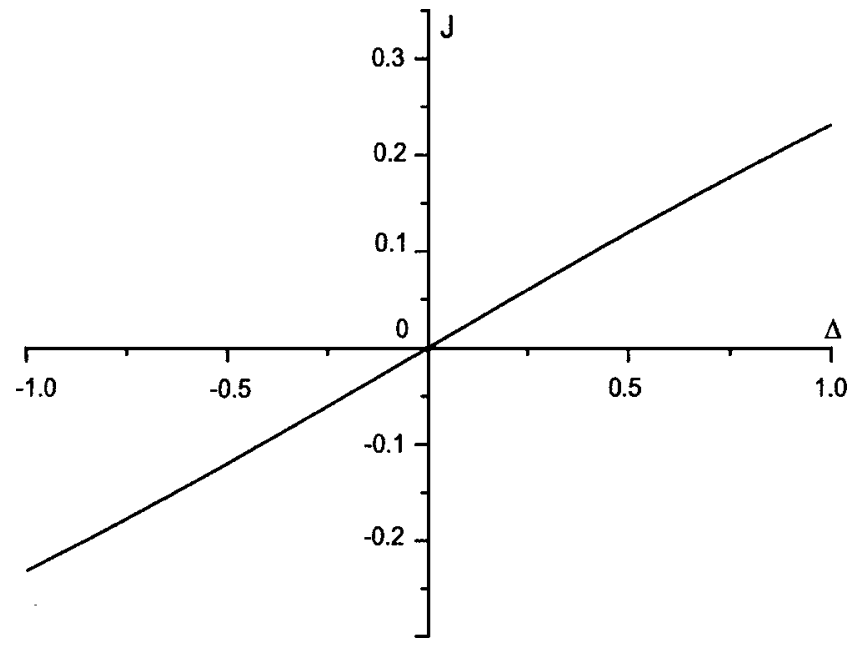

FIG. 2. Current $J$ versus asymmetric parameter $\Delta$ of the potential at $U_{0}=5, F_{0}=3.0, L=1.0, T=1.0$, and $\delta=0$.

$$
\begin{gathered}
B=\frac{L^{2}-\Delta^{2}}{4 a b T}\left(1-e^{-a}\right)\left(e^{b}-1\right), \\
C=\frac{1}{T}\left(\frac{L-\Delta}{2 b}\right)^{2}\left(e^{b}-1-b\right), \\
a=\frac{2 U_{0}-F(t)(L+\Delta)}{2(T+\delta)}, \\
b=\frac{2 U_{0}+F(t)(L-\Delta)}{2 T} .
\end{gathered}
$$

The average current is

$$
J=\frac{1}{\tau} \int_{0}^{\tau} j(F(t)) d t
$$

where $\tau$ is the period of the driving force $F(t) . \tau$ is assumed to be longer than any other time scale of the system at quasisteady-state. For the external force $F(t)$ shown in Fig. 1(b),

$$
J=\frac{1}{2}\left[j\left(F_{0}\right)+j\left(-F_{0}\right)\right] .
$$

When both the potential and the temperature are symmetric $\left(\delta=0, F_{0}=0\right)$, the current $J$ reduces to

$$
J=\frac{1}{2(2 T+\delta)}\left(\frac{U_{0}}{2 L}\right)^{2}\left(\frac{1}{e^{U_{0} /(T+\delta)}-1}-\frac{1}{e^{U_{0} / T}-1}\right) .
$$

Therefore, the net current is not zero even when both the potential and the temperature are spatially symmetric. The direction of the current is determined by the sign of $\delta$. The particle tends to move from the high- to the low-temperature region. In fact, this agrees with the diffuse law.

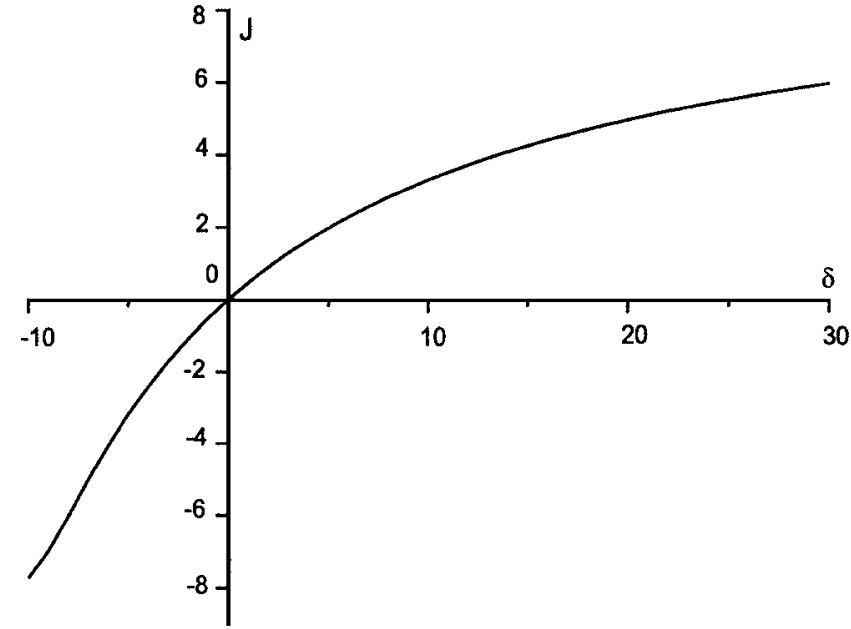

FIG. 3. Current $J$ versus temperature difference $\delta$ at $U_{0}=5$, $F_{0}=3.0, L=1.0, T=10$, and $\Delta=0$.

\section{RESULTS AND DISCUSSION}

Figure 2 shows the current $J$ as a function of the asymmetric parameter $\Delta$ of the potential at $\delta=0$. The current is negative for $\Delta<0$, zero at $\Delta=0$, and positive for $\Delta>0$. Therefore, we can have the current reversal by changing the sign of $\Delta$, the asymmetry of the potential.

Figure 3 shows the current $J$ versus temperature difference $\delta$ in a symmetric potential $\Delta=0$. The temperature difference $\delta$ controls not only the magnitude but also the direction of the current. When $\delta=0$ and $\Delta=0$, there is no current. For asymmetric potentials, varying the temperature difference is another way of inducing a net current.

The current $J$ as a function of $T$ is shown in Fig. 4 for different combinations of $\Delta$ and $\delta$. The curve is observed to be bell shaped, which shows the feature of resonance. When $T \rightarrow 0, J$ tends to zero for all values of $\delta$ and $\Delta$. Therefore, the particle cannot pass the barrier and there is no current. When $T \rightarrow \infty$ so that the thermal noise is very large, the ratchet effect disappears and $J \rightarrow 0$, also. There is an opti-

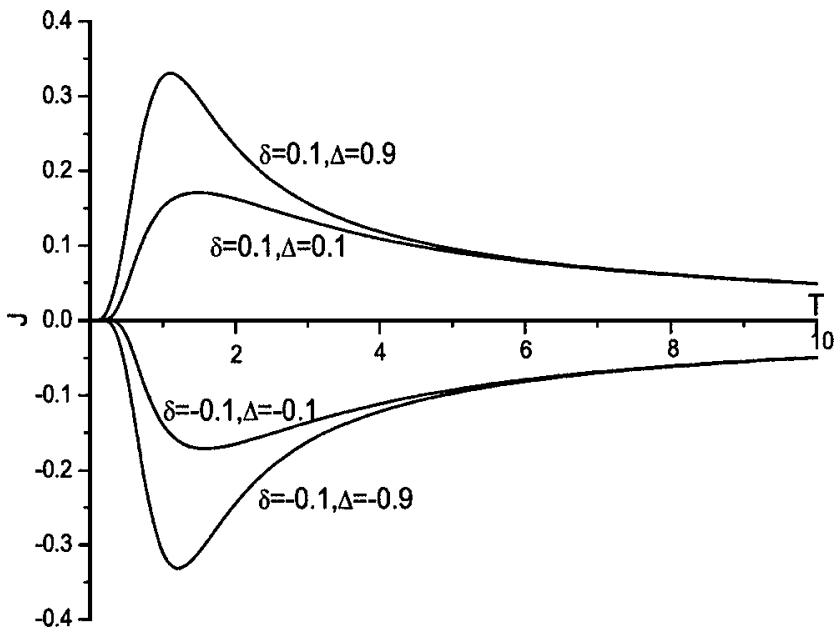

FIG. 4. Current $J$ versus temperature $T$ for different asymmetric parameters $\delta$ and $\Delta$ at $U_{0}=5, F_{0}=3.0$, and $L=1.0$. 

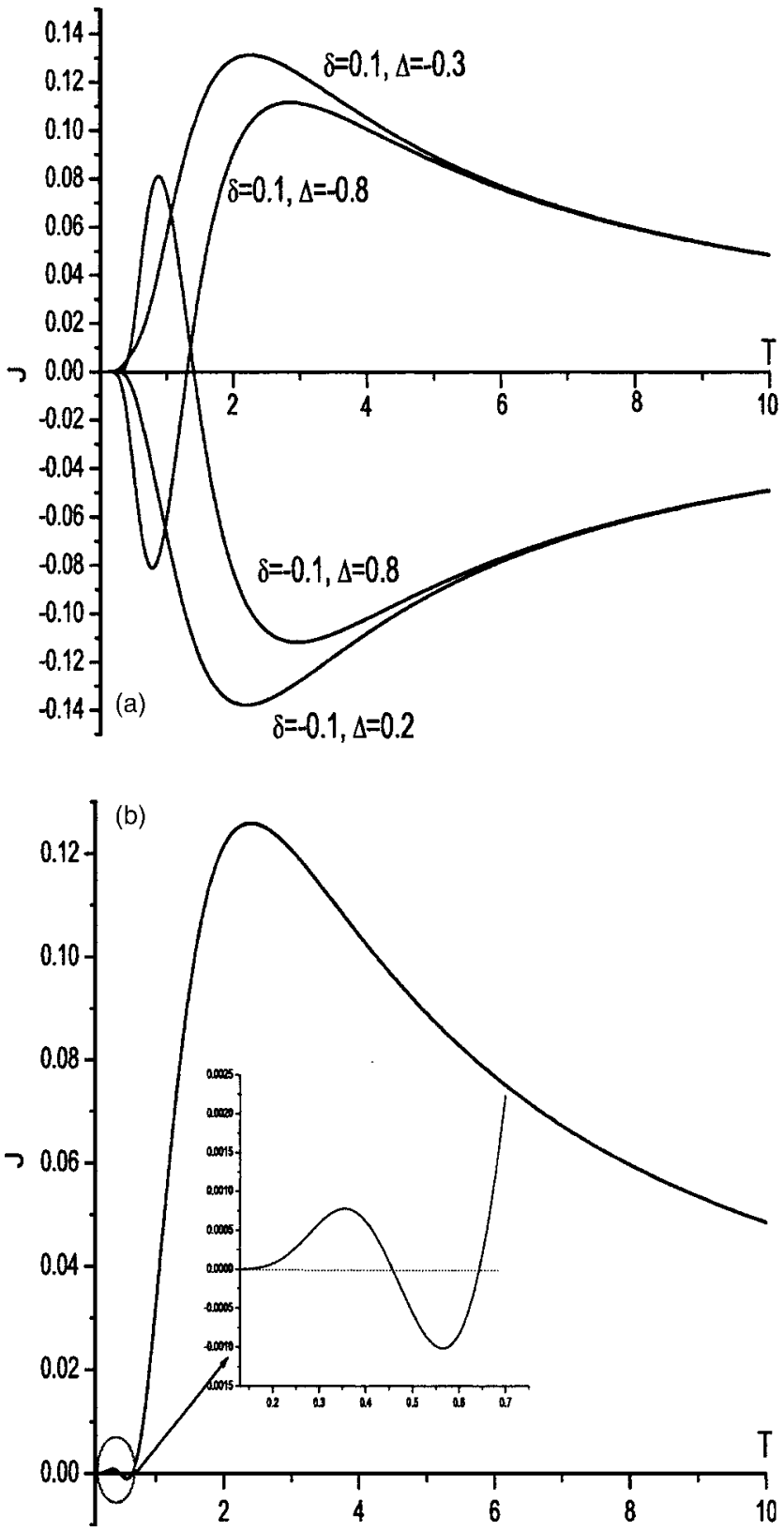

FIG. 5. (a) Current $J$ versus temperature $T$ for different values of the asymmetric parameters $\Delta$ at $U_{0}=5, F_{0}=3.0, L=1.0$. (b) Current $J$ versus temperature $T$ at $U_{0}=5, F_{0}=3.0, L=1.0, \delta=0.1$, and $\Delta=-0.4$.

mized value of $T$ at which the current $J$ takes its maximum value. There is no current reversal at $\delta=0.1, \Delta=0.9 ; \delta=0.1$, $\Delta=0.1 ; \delta=-0.1, \Delta=-0.1 ;$ and $\delta=-0.1, \Delta=-0.9$. In fact, the temperature cannot lead to current reversal if $\Delta \delta>0$ (Figs. 2-4).

In Fig. 5(a), we plot the current $J$ as a function of temperature $T$ for different combinations of $\Delta$ and $\delta$. When the temperature difference $(\delta=0.1)$ is positive, the current may reverse its direction on increasing temperature for negative $\Delta(\Delta=-0.8)$. It is observed that the current reversal may occur for negative $\delta$ and positive $\Delta(\delta=-0.1, \Delta=0.8)$. We can also have the current reversal twice at $\delta=0.1, \Delta=-0.4$ [Fig. 5(b)]. Therefore, there may exist current reversal

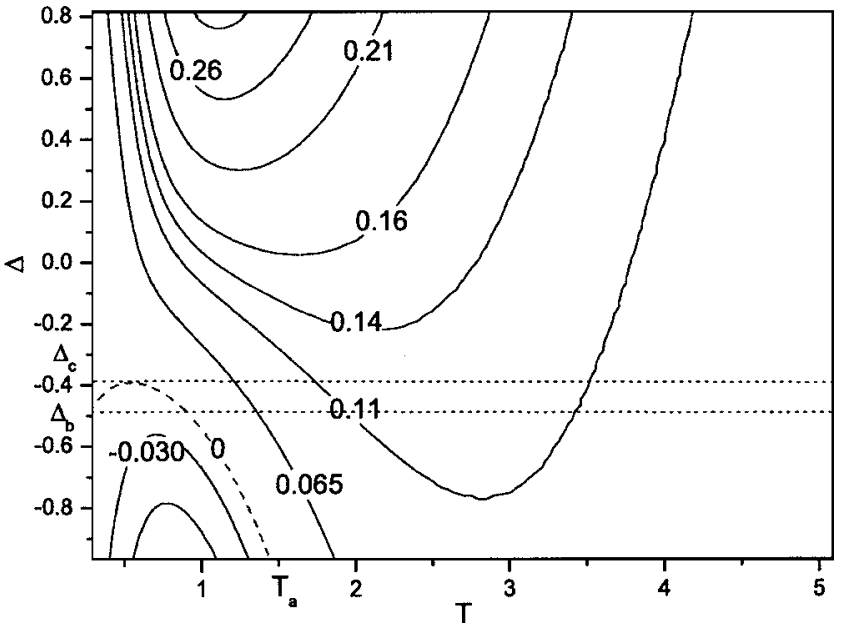

FIG. 6. Current contours on the $\Delta-T$ plane at $U_{0}=5, F_{0}=3.0$, $L=1.0$, and $\delta=0.1: \Delta_{c}(-0.3987)$ is the maximum $\Delta$ for the curve $J=0 ; \Delta_{b}(-0.4913)$ is the asymmetric parameter of the potential at the crossing point of $J=0$ and $T=0 ; T_{a}$ is the temperature at the crossing point of $J=0$ and $\Delta=-1.0$.

for $\Delta \delta<0$. However, $\Delta \delta<0$ is not a sufficient condition for current reversal. For example, the current is always positive for $\delta=0.1, \Delta=-0.3$ and negative for $\delta=-0.1, \Delta=0.2$ [Fig. 5(a)].

In order to illustrate the current reversal in detail, the current contours are shown in Figs. 6 and 7, respectively. When $T>T_{a}$ or $\Delta>\Delta_{c}$, the current is always positive; there is no current reversal [Fig. 6; also see the case $\delta=0.1$, $\Delta=-0.3$ in Fig. 5(a)]. The current reversal may, however, occur by varying $T$ or $\Delta$ when $T<T_{a}$ or $\Delta<\Delta_{c}$ [Fig. 6; also see the case $\delta=0.1, \Delta=-0.8$ in Fig. 5(a) ]. In particular, the current may reverse its direction twice if $\Delta_{b}<\Delta<\Delta_{c}$ [Fig. 6; also see the case $\delta=0.1, \Delta=-0.4$ in Fig. 5(b)].

The current is always negative and there is no current reversal for $\delta \leqslant 0$ and $\Delta=-0.6$ (Fig. 7). When $\delta_{a}<\delta<\delta_{c}$, the current may reverse its direction on increasing temperature.

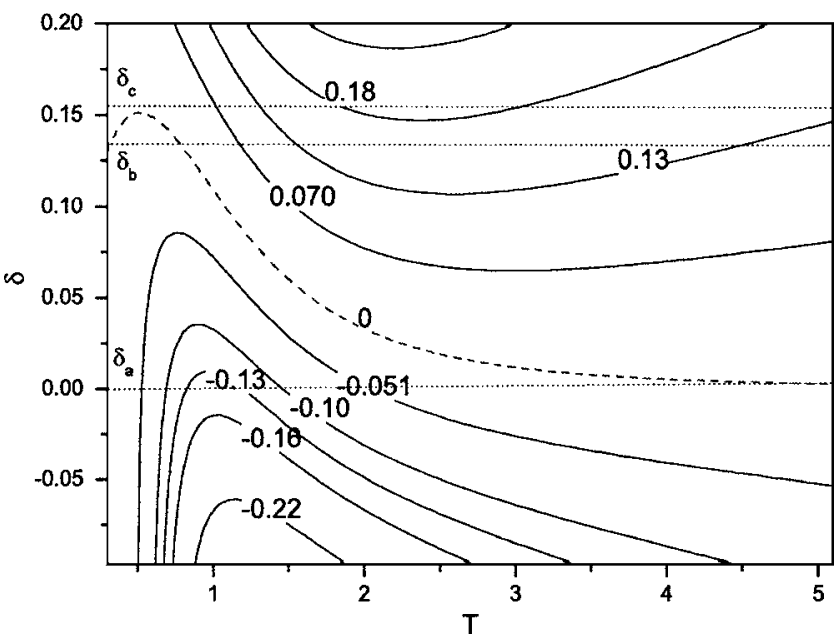

FIG. 7. Current contours on the $\delta$ - $T$ plane at $U_{0}=5, F=3.0$, $L=1.0$, and $\Delta=-0.6: \delta_{a}=0 ; \delta_{b}(0.138)$ is the temperature difference at the crossing point of $J=0$ and $T=0 ; \delta_{c}(0.151)$ is the maximum temperature difference for $J=0$. 
The current may change its direction twice, in particular, when $\delta_{b}<\delta<\delta_{c}$.

Therefore, we cannot have current reversal when $\delta \Delta \geqslant 0$. When $\delta \Delta<0$, the current may reverse its direction. However, $\delta \Delta<0$ is not a sufficient condition for current reversal.

\section{CONCLUDING REMARKS}

The transport of a Brownian particle moving in a periodic potential is studied in the presence of an unbiased fluctuation and two heat reservoirs. In the quasi-steady-state limit, we obtain the current analytically. It is found that the asymmetric parameter $\Delta$ of the potential and the temperature difference $\delta$ are the two pivotal factors for obtaining a net current. For two positive or two negative driving factors such that $\Delta \delta>0$, the current cannot reverse its direction. Current reversal cannot occur either if there is only one driving factor such that $\Delta \delta=0$. For two opposite driving factors so that $\Delta \delta<0$, current reversal may occur. Current reversal can also occur twice in certain conditions. The condition $\Delta \delta<0$ is a necessary but not a sufficient condition for current reversal.
[1] J. L. Mateos, Phys. Rev. Lett. 84, 258 (2000).

[2] J. Luczka, R. Bartussek, and P. Hanggi, Europhys. Lett. 31, 431 (1995).

[3] L. P. Faucheux et al., Phys. Rev. Lett. 74, 1504 (1995).

[4] B. Q. Ai, X. J. Wang, G. T. Liu, and L. G. Liu, Phys. Rev. E 68, 061105 (2003); 67, 022903 (2003); B. Q. Ai, G. T. Liu, H. Z. Xie, and L. G. Liu, Chaos 14, 957 (2004).

[5] J. Maddox, Nature (London) 368, 287 (1994); R. D. Astumian and I. Derenyi, Eur. Biophys. J. 27, 474 (1998); N. Thomas and R. A. Thornhill, J. Phys. D 31, 253 (1998); C. R. Doering, B. Ermentrout, and G. Oster, Biophys. J. 69, 2256 (1995).

[6] J. Krim, D. H. Solina, and R. Chiarello, Phys. Rev. Lett. 66, 181 (1991); J. B. Sokolov, J. Krim, and A. Widom, Phys. Rev. B 48, 9134 (1993); L. Daikhin and M. Urbakh, Phys. Rev. E 49, 1424 (1994); C. Daly and J. Krim, Phys. Rev. Lett. 76, 803 (1996); M. R. Sorensen, K. W. Jacobsen, and P. Stoltze, Phys. Rev. B 53, 2101 (1996).

[7] I. Derenyi, C. Lee, and A.-L. Barabasi, Phys. Rev. Lett. 80, 1473 (1998).

[8] I. Zapata, R. Bartussek, E. Sols, and P. Hanggi, Phys. Rev. Lett. 77, 2292 (1996).

[9] L. P. Faucheux, L. S. Bourdieu, P. D. Kaplan, and A. J. Libchaber, Phys. Rev. Lett. 74, 1504 (1995); C. MenneratRobilliard, D. Lucas, S. Guibal, J. Tabosa, C. Jurczak, J.-Y. Courtois, and G. Grynberg, ibid. 82, 851 (1999).

[10] L. Gorre-Talini, J. P. Spatz, and P. Silberzan, Chaos 8, 650 (1998); I. Derenyi and R. D. Astumian, Phys. Rev. E 58, 7781 (1998); D. Ertas, Phys. Rev. Lett. 80, 1548 (1998); T. A. J. Duke and R. H. Austin, ibid. 80, 1552 (1998).

[11] M. O. Magnasco, Phys. Rev. Lett. 71, 1477 (1993).

[12] P. Hanggi and R. Bartussek, Nonlinear Physics of Complex Systems-Current Status and Future TrendsLecture notes in Physics 476 (Springer, Berlin, 1996), p. 294.

[13] P. Reimann, R. Bartussek, R. Haussler, and P. Hanggi, Phys. Lett. A 215, 26 (1994).
[14] C. R. Doering, W. Horsthemke, and J. Riordan, Phys. Rev. Lett. 72, 2984 (1994).

[15] C. Kettner, Phys. Rev. E 61, 312 (2000).

[16] R. Tammelo, R. Mankin, and D. Martila, Phys. Rev. E 66, 051101 (2002).

[17] D. Dan, M. C. Mahato, and A. M. Jayannavar, Phys. Rev. E 63, 056307 (2001).

[18] B. Q. Ai, X. J. Wang, G. T. Liu, H. Z. Xie, D. H. Wen, W. Chen, and L. G. Liu., Eur. Phys. J. B 37, 523 (2004).

[19] M. M. Millonas and M. I. Dykman, Phys. Lett. A 185, 65 (1994).

[20] J.-F. Chauwin, A. Ajdari, and J. Prost, Europhys. Lett. 32, 373 (1995).

[21] M. Bier and R. D. Astumian, Phys. Rev. Lett. 76, 4277 (1996).

[22] C. R. Doering, W. Horsthemke, and J. Riordan, Phys. Rev. Lett. 72, 2984 (1994).

[23] R. Bartussek, P. Reimann, and P. Hanggi, Phys. Rev. Lett. 76, 1166 (1996).

[24] R. Mankin, A. Ainsaar, A. Haljas, and E. Reiter, Phys. Rev. E 63, 041110 (2001).

[25] J. Kula, T. Czernik, and J. Luczka, Phys. Rev. Lett. 80, 1377 (1998).

[26] M. Kostur and J. Luczka, Phys. Rev. E 63, 021101 (2001).

[27] I. Derenyi and A. Ajdari, Phys. Rev. E 54, R5 (1996).

[28] F. Marchesoni, Phys. Lett. A 237, 126 (1998); H. A. Larrondoa, Fereydoon Family, and C. M. Arizmendia, Physica A 303, 67 (2002).

[29] G. Oster and H. Wang, in Molecular Motors, edited by M. Schliwa (Wiley-VCH, Weinheim, 2002), pp. 207-228.

[30] H. Risken, The Fokker-Planck Equation (Springer-Verlag, Berlin, 1984).

[31] M. Asfaw and M. Bekele, Eur. Phys. J. B 38, 457 (2004).

[32] U. Henningsen and M. Schliwa, Nature (London) 389, 93 (1997). 